\title{
Multichannel Image Restoration Based on Optimization of the Structural Similarity Index
}

\author{
Maja Temerinac-Ott ${ }^{* \dagger}$ and Hans Burkhardt ${ }^{* \dagger}$ \\ * Institute of Computer Science, University of Freiburg, \\ Chair of Pattern Recognition and Image Processing, Georges-Köhler-Allee Geb. 052, \\ 79110 Freiburg, Germany, temerina@informatik.uni-freiburg.de \\ ${ }^{\dagger}$ Centre for Biological Signalling Studies (bioss), University of Freiburg, Germany
}

\begin{abstract}
In this paper a framework for multichannel image restoration based on optimization of the structural similarity (SSIM) index is presented. The SSIM index describes the similarity of images more appropriately for the human visual system than the mean square error (MSE). It has not yet been explored for the multi channel restoration task. The construction of an optimization algorithm is difficult due to the non-linearity of the SSIM measure. The existing solution based on a quasi-convex problem formulation is successfully extended for the multichannel image restoration. The correctness of the algorithm is verified on sample images and it is shown that multi-view information can significantly improve the restoration results.
\end{abstract}

Keywords-multichannel image restoration; structural similarity; inverse filter; quasi-convex optimization of non-linear functions

\section{INTRODUCTION}

The structural similarity (SSIM) index has received quite a few attention in recent publications ([1],[2],[3]). Due to its ability to capture the similarity of two images in a more appropriate way than the mean square error (MSE), it poses a real alternative to comparing images. The SSIM index computes a product between three measures: luminance, contrast and structural correlation. These measures seem to capture the human visual perception in a realistic way.

Multichannel processing of images is used whenever multi images of the same scene are available. This occurs for example when a moving object is recorded several times with the same camera, e.g. the license plate of a driving car, and the license number needs to be reconstructed. Another example where this kind of reconstruction is needed, is in recording biological objects with a microscope from different viewing angles. The goal is to use the multiinformation present in those images in order to restore the original image more appropriately.

Several multichannel restoration methods based on MSE ([4], [5], [7]) have already been developed. In this work, a framework for multiview restoration based on the SSIM index is designed. The work by Channappayya et al ([3]) is extended to multichannels.

\section{A. Problem Outline}

The recording of an image $\mathrm{x}$ can be described by the point spread function $\mathbf{h}$ and the noise $\eta$ introduced by the recording system. The recording process can be approximated by a wide sense stationary process:

$$
\mathbf{y}=\mathbf{h} * \mathbf{x}+\eta
$$

where $*$ is the convolution operator.

Additive gaussian noise with standard deviation $\sigma_{x}$ is assumed in our model. The goal of multi-channel restoration is to find the best estimate of $\hat{\mathrm{x}}$ given several recorded images $\mathbf{y}_{i}$. The quality of the reconstructed image $\hat{\mathbf{x}}$ is measured by a similarity measure. The simplified form of the SSIM index as defined by [1] is chosen as the similarity measure:

$$
\operatorname{SSIM}(\mathbf{x}, \hat{\mathbf{x}})=\frac{2 \mu_{\mathbf{x}} \mu_{\hat{\mathbf{x}}}+C_{1}}{\mu_{\mathbf{x}}^{2}+\mu_{\hat{\mathbf{x}}}^{2}+C_{1}} \cdot \frac{2 \sigma_{\mathbf{x} \hat{\mathbf{x}}}+C_{2}}{\sigma_{\mathbf{x}}^{2}+\sigma_{\hat{\mathbf{x}}}^{2}+C_{2}},
$$

where $C_{1}$ and $C_{2}$ are stabilizing constants, $\mu_{\mathrm{x}}$ is the mean value and $\sigma_{\mathbf{x}}^{2}$ the variance.

\section{B. Related Work}

In [3] the statistical SSIM index has been introduced and the restoration problem was solved for single channel images. This solution is extended for multi-channel images. The statistical SSIM index is a straight forward extension of the SSIM index by raplacing the empirical properties by their statistical equivalences:

$$
\begin{array}{rr}
\mu_{\mathbf{x}}= & E[\mathbf{x}[n]] \\
\sigma_{\mathbf{x}}^{2}= & E\left[\left(\mathbf{x}[n]-\mu_{\mathbf{x}}\right)^{2}\right] \\
\sigma_{\mathbf{x y}}= & E\left[\left(\mathbf{x}[n]-\mu_{\mathbf{x}}\right)\left(\mathbf{y}[n]-\mu_{\mathbf{y}}\right)\right]
\end{array}
$$

\section{Notation}

Bold letters are used to denote vectors, e.g. $\mathrm{g}=$ $(g[0], g[1], \ldots, g[N-1])^{T}$ and $\mathbf{e}=(1,1, \ldots, 1)^{T}$ are both length $N$ vectors. The mean of the source and the observed object are $\mu_{\mathbf{x}}$ and $\mu_{\mathbf{y}}$. The cross covariance between the source $(x[n])$ and the observed image $\mathbf{y}=(y[n], y[n-$ $1], \ldots, y[n-(N-1)])^{T}$ is defined by $c_{\mathbf{x y}}=E[(x[n]-$ 


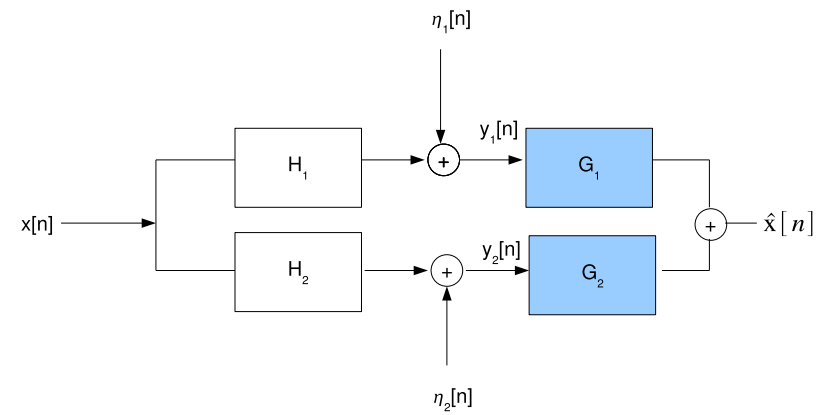

Figure 1. Block diagram. The inverse filter $\mathbf{g}_{1}, \ldots, \mathbf{g}_{\mathbf{M}}$ are optimized adjointly to produce the best reconstruction of image $\mathbf{x}$ according to the SSIM index.

$\left.\left.\mu_{\mathbf{x}}\right)\left(\mathbf{y}-\mathbf{e} \mu_{\mathbf{y}}\right)\right]$ and $\mathbf{K}_{\mathbf{y y}}=E\left[\left(\mathbf{y}-\mathbf{e} \mu_{\mathbf{y}}\right)\left(\mathbf{y}-\mathbf{e} \mu_{\mathbf{y}}\right)\right]$ is the covariance matrix of size $N \times N$.

\section{Paper Organization}

In Section II the framework for multichannel restoration for $\mathrm{M}$ images is described and a general solution for finding the inverse filters is presented. In Section III, the solution for the case of two images is computed explicitly. In Section IV experiments based on the solution in Section III are presented. In Section $\mathrm{V}$ a conclusion is drawn and future research are discussed.

\section{Problem Formulation}

The following model(See Figure 1) is considered: Image $\mathbf{x}$ is recorded $M$ times and the resulting images are $\mathbf{y}_{1}, \ldots, \mathbf{y}_{\mathbf{M}}$. The main goal is to find inverse filters $\mathbf{g}_{\mathbf{1}}, . ., \mathbf{g}_{\mathbf{M}}$ in order to restore the image, given the images $\mathbf{y}_{\mathbf{1}}, \ldots, \mathbf{y}_{\mathbf{M}}$, the blurring filters $H_{1}, \ldots, H_{M}$ and the probability density function (PDF) of the noise.

The restored image $\hat{\mathbf{x}}$ is then computed by:

$$
\hat{\mathbf{x}}[n]=\mathbf{g}_{\mathbf{1}}[n] * \mathbf{y}_{\mathbf{1}}[n]+\ldots+\mathbf{g}_{\mathbf{M}}[n] * \mathbf{y}_{\mathbf{M}}[n] .
$$

The inverse filter $\mathbf{g}_{\mathbf{1}}, \ldots, \mathbf{g}_{\mathbf{M}}$ of size $N$ are found adjointly by optimizing the statistical SSIM index (for a definition see [3]) between the original image $\mathbf{x}$ and the restored image $\hat{\mathbf{x}}$ :

$$
\hat{\mathbf{g}}=\arg \max _{\mathbf{g} \in \mathbb{R}^{M N}} \operatorname{StatSSIM}(\mathbf{x}[n], \hat{\mathbf{x}}[n]) .
$$

The StatSSIM is computed explicitly by:

$$
\begin{aligned}
\operatorname{StatSSIM}(\mathbf{x}[n], \hat{\mathbf{x}}[n]) & =\frac{2 \mu_{\mathbf{x}} \mu_{\hat{\mathbf{x}}}+C_{1}}{\mu_{\mathbf{x}}^{2}+\mu_{\hat{\mathbf{x}}}^{2}+C_{1}} \cdot \frac{2 \sigma_{\mathbf{x} \hat{\mathbf{x}}}+C_{2}}{\sigma_{\mathbf{x}}^{2}+\sigma_{\hat{\mathbf{x}}}^{2}+C_{2}} \\
& =
\end{aligned}
$$

$$
\begin{aligned}
\mathbf{Q}_{\mathbf{1}} & =\frac{2 \mu_{\mathbf{x}} E\left[\sum_{k=1}^{M} \sum_{i=0}^{N-1} \mathbf{g}_{\mathbf{k}}[i] \mathbf{y}_{\mathbf{k}}[n-i]\right]+C_{1}}{\mu_{\mathbf{x}}^{2}+\left(E\left[\sum_{k=1}^{M} \sum_{i=0}^{N-1} \mathbf{g}_{\mathbf{k}}[i] \mathbf{y}_{\mathbf{k}}[n-i]\right]\right)^{2}+C_{1}} \\
& =\frac{2 \mu_{\mathbf{x}}\left(\mathbf{g}_{\mathbf{1}}^{\mathbf{T}} \mathbf{e} \mu_{\mathbf{y}_{\mathbf{1}}}+\ldots+\mathbf{g}_{\mathbf{M}} \mathbf{T} \mathbf{e} \mu_{\mathbf{y}_{\mathbf{M}}}\right)+C_{1}}{\mu_{\mathbf{x}}^{2}+\left(\mathbf{g}_{\mathbf{1}}^{\mathbf{T}} \mathbf{e} \mu_{\mathbf{y}_{\mathbf{1}}}+\ldots+\mathbf{g}_{\mathbf{M}} \mathbf{T} \mathbf{e} \mu_{\mathbf{y}_{\mathbf{M}}}\right)^{2}+C_{1}} \\
\mathbf{Q}_{\mathbf{2}}= & \frac{2 E\left[\left(\mathbf{x}[n]-\mu_{\mathbf{x}}\right)\left(\sum_{k=1}^{M} \sum_{i=0}^{N-1}\left(\mathbf{g}_{\mathbf{k}}[i]-\mu_{\mathbf{y}_{\mathbf{k}}}\right)\right]+C_{2}\right.}{E\left[\left(\mathbf{x}[n]-\mu_{\mathbf{x}}\right)^{2}\right]+E\left[\left(\sum_{k=1}^{M} \sum_{i=0}^{N-1} \mathbf{g}_{\mathbf{k}}[i]-\mu_{\mathbf{y}_{\mathbf{k}}}\right)^{2}\right]+C_{2}} \\
= & \frac{2 \sum_{i=1}^{M} \mathbf{g}_{\mathbf{i}} \mathbf{T} \mathbf{c}_{\mathbf{x} \mathbf{y}_{\mathbf{i}}}+C_{2}}{\sigma_{\mathbf{x}}^{2}+2 \sum_{i=1}^{M} \sum_{j=1}^{M} \mathbf{g}_{\mathbf{i}} \mathbf{T}_{\mathbf{y}_{\mathbf{i}} \mathbf{y}_{\mathbf{j}}} \mathbf{g}_{\mathbf{j}}+C_{2}}
\end{aligned}
$$

where $\mathbf{K}_{\mathbf{y y}}$ is the covariance matrix and $\mathbf{c}_{\mathbf{x y}}$ the cross covariance vector between the source $\mathbf{x}$ and the observed process $\mathbf{y}$.

A nonlinear equation is obtained consisting of the multiplication of two quotients. The first quotient depends only on $\mathbf{g}_{\mathbf{i}}$. By constraining $\mathbf{g}_{\mathbf{i}}^{\mathbf{T}} \mathbf{e}$ to $\alpha_{i}$, the optimization problem is simplified to finding $\hat{\mathbf{g}}(\alpha)$ :

$$
\begin{gathered}
\quad \hat{\mathbf{g}}(\alpha)=\arg \max _{\mathbf{g} \in \mathbb{R}^{M N}} \mathbf{Q}_{\mathbf{2}} \\
\text { subject to: } \mathbf{g}^{T} \mathbf{e}=\alpha,
\end{gathered}
$$

where $\mathbf{g}$ is a matrix with the rows being the vectors $g_{1}, \ldots g_{M}$. Following [3], a boundary $\gamma$ to $\operatorname{StatSSIM}(\mathbf{x}[n], \hat{\mathbf{x}}[n])$ is set and the above equation is converted to a quasi-complex optimization problem:

$\min : \gamma$

subject to:

$$
\max : \mathbf{Q}_{\mathbf{2}} \leq \gamma
$$$$
\text { subject to: } \mathbf{g}^{T} \mathbf{e}=\alpha \text {. }
$$

$f(\gamma)$ is defined by:

$$
\begin{array}{r}
f(\gamma)=\gamma\left(\sigma_{\mathbf{x}}^{2}+2 \sum_{i=1}^{M} \sum_{j=1}^{M} \mathbf{g}_{\mathbf{i}}{ }^{\mathbf{T}} \mathbf{K}_{\mathbf{y}_{\mathbf{i}} \mathbf{y}_{\mathbf{j}}} \mathbf{g}_{\mathbf{j}}+C_{2}\right) \\
-\left(2 \sum_{i=1}^{M} \mathbf{g}_{\mathbf{i}}^{\mathbf{T}} \mathbf{c}_{\mathbf{x} \mathbf{y}_{\mathbf{i}}}+C_{2}\right)
\end{array}
$$

and equation (8) is reformulated as:

$\min : \gamma$

subject to:

$$
\min : f(\gamma) \geq 0
$$$$
\text { subject to: } \mathbf{g}^{T} \mathbf{e}=\alpha \text {. }
$$

The overall problem is convex and can be solved by applying the Lagrange multipliers $\lambda$ and applying the first order sufficiency condition:

$$
\nabla \mathbf{g}\left(f(\gamma)+\lambda_{1}\left(g_{1}^{T} e-\alpha_{1}\right)+\ldots+\lambda_{M}\left(g_{M}^{T} e-\alpha_{M}\right)\right)=0
$$

A set of linear equations is obtained and can be solved easily. In Section III, an explicit solution for $M=2$ is presented.

The optimal $\gamma$ is computed using the bisection method as proposed in [3]: 
1. Initialize $\gamma\left(\right.$ say $\left.\gamma_{0}\right)$ between 0 and 1 .

Set upLimit $=1$, lowLimit $=\gamma_{0}$

2. Evaluate the optimal filter.

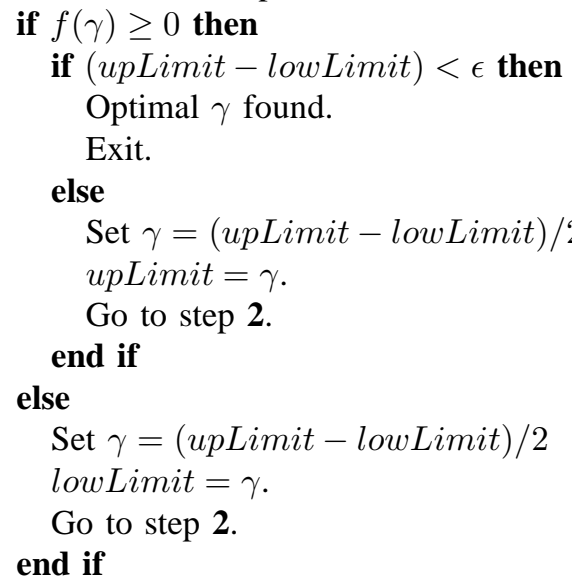

Algorithm 1. Algorithm to search for the optimal $\gamma$

\section{SOLUTION FOR Two IMAGES}

The gradient $\nabla \mathbf{g}_{1}, \nabla \mathbf{g}_{2}, \nabla \lambda_{1}$ and $\nabla \lambda_{2}$ is computed:

$$
\begin{aligned}
& \nabla \mathbf{g}_{1}\left(f(\gamma)+\lambda_{1}\left(g_{1}^{T} e-\alpha_{1}\right)+\lambda_{2}\left(g_{2}^{T} e-\alpha_{2}\right)\right) \\
& =\gamma\left(2 \mathbf{K}_{\mathbf{y}_{\mathbf{1}} \mathbf{y}_{\mathbf{1}}} \mathbf{g}_{\mathbf{1}}+2 \mathbf{K}_{\mathbf{y}_{1} \mathbf{y}_{\mathbf{2}}} \mathbf{g}_{\mathbf{2}}-2 \mathbf{c}_{\mathbf{x y}_{\mathbf{1}}}+\lambda_{1} \mathbf{e}\right)=0 \\
& \nabla \mathbf{g}_{2}\left(f(\gamma)+\lambda_{1}\left(g_{1}^{T} e-\alpha_{1}\right)+\lambda_{2}\left(g_{2}^{T} e-\alpha_{2}\right)\right) \\
& =\gamma\left(2 \mathbf{K}_{\mathbf{y}_{2} \mathbf{y}_{1}} \mathbf{g}_{1}+2 \mathbf{K}_{\mathbf{y}_{2} \mathbf{y}_{2}} \mathbf{g}_{2}-2 \mathbf{c}_{\mathbf{x y}_{2}}+\lambda_{2} \mathbf{e}\right)=0 \\
& \nabla \lambda_{1}\left(f(\gamma)+\lambda_{1}\left(g_{1}^{T} e-\alpha_{1}\right)+\lambda_{2}\left(g_{2}^{T} e-\alpha_{2}\right)\right) \\
& =\mathbf{g}_{1}^{\mathbf{T}} \mathbf{e}-\alpha_{1}=0 \\
& \nabla \lambda_{2}\left(f(\gamma)+\lambda_{1}\left(g_{1}^{T} e-\alpha_{1}\right)+\lambda_{2}\left(g_{2}^{T} e-\alpha_{2}\right)\right) \\
& =\mathbf{g}_{2}^{\mathbf{T}} \mathbf{e}-\alpha_{2}=0
\end{aligned}
$$

The gradient equations (12) and (13) are rewritten by:

$$
\begin{aligned}
& \mathbf{A g}_{1}+\mathbf{B g}_{2}=\mathbf{h}_{1} \\
& \mathbf{B}^{\mathrm{T}} \mathbf{g}_{\mathbf{1}}+\mathbf{C g}_{\mathbf{2}}=\mathbf{h}_{\mathbf{2}},
\end{aligned}
$$

where $\mathbf{A}, \mathbf{B}, \mathbf{C}, \mathbf{h}_{\mathbf{1}}, \mathbf{h}_{\mathbf{2}}$ are defined as:

$$
\begin{aligned}
& \mathbf{A}:=2 \gamma \mathbf{K}_{\mathbf{y}_{1} \mathbf{y}_{\mathbf{1}}} \\
& \mathbf{B}:=2 \gamma \mathbf{K}_{\mathbf{y}_{1} \mathbf{y}_{\mathbf{2}}} \\
& \mathbf{C}:=2 \gamma \mathbf{K}_{\mathbf{y}_{\mathbf{2}} \mathbf{y}_{\mathbf{2}}} \\
& \mathbf{h}_{1}:=2 \mathbf{c}_{\mathbf{x y}_{1}}-\lambda_{1} \mathbf{e} \\
& \mathbf{h}_{\mathbf{2}}:=2 \mathbf{c}_{\mathbf{x y}_{\mathbf{2}}}-\lambda_{2} \mathbf{e}
\end{aligned}
$$

From (16) follows:

$$
\mathrm{g}_{1}=\mathbf{A}^{-1}\left(\mathbf{h}_{1}-\mathbf{B g}_{2}\right)
$$

and substitute it in :

$$
\mathbf{B}^{\mathrm{T}}\left(\mathbf{A}^{-1}\left(\mathbf{h}_{1}-\mathbf{B g}_{2}\right)\right)+\mathbf{C g}_{2}=\mathbf{h}_{2}
$$

Now the equation is solved for $\mathbf{g}_{2}$ :

$$
\mathrm{g}_{2}=\mathrm{g}_{2,0}+\lambda_{1} \mathrm{~g}_{2,1}+\lambda_{2} \mathbf{g}_{2,2},
$$

with

$$
\begin{array}{r}
\mathbf{g}_{\mathbf{2}, \mathbf{0}}:=\frac{1}{\gamma} \mathbf{K}^{-\mathbf{1}} \mathbf{c} \\
\mathbf{g}_{2, \mathbf{1}}:=\frac{1}{2 \gamma} \mathbf{K}^{-\mathbf{1}} \mathbf{K}_{\mathbf{y}_{\mathbf{2}} \mathbf{y}_{\mathbf{1}}} \mathbf{K}_{\mathbf{y}_{1} \mathbf{y}_{\mathbf{1}}}^{-\mathbf{e}} \mathbf{} \\
\mathbf{g}_{\mathbf{2}, \mathbf{2}}:=\frac{1}{2 \gamma} \mathbf{K}^{-\mathbf{1}} \mathbf{e}
\end{array}
$$

where $\mathbf{K}$ and $\mathbf{c}$ are defined as:

$$
\begin{array}{r}
\mathrm{K}:=\mathrm{K}_{\mathrm{y}_{2} \mathrm{y}_{2}}-\mathrm{K}_{\mathrm{y}_{2} \mathrm{y}_{1}} \mathbf{K}_{\mathrm{y}_{1} \mathrm{y}_{1}}^{-1} K_{\mathrm{y}_{1} \mathrm{y}_{2}} \\
\mathbf{c}:=\mathbf{c}_{\mathrm{xy}_{2}}-\mathbf{K}_{\mathrm{y}_{2} \mathrm{y}_{1}} \mathbf{K}_{\mathrm{y}_{1} \mathrm{y}_{1}}^{-1} \mathbf{c}_{\mathrm{x}_{1}}
\end{array}
$$

Now $\mathbf{g}_{2}$ from (21) is substituted to equation (19) and the equation is rewritten by:

$$
\mathbf{g}_{1}=\mathbf{g}_{1,0}+\lambda_{1} \mathbf{g}_{1,1}+\lambda_{2} \mathbf{g}_{1,2},
$$

with

$$
\begin{array}{r}
\mathbf{g}_{1,0}:=\frac{1}{\gamma}\left(\mathbf{K}_{\mathbf{y}_{1} \mathbf{y}_{1}}^{-1}\left(\mathbf{c}_{\mathbf{x y}_{1}}-\mathbf{K}_{\mathbf{y}_{1} \mathbf{y}_{2}} \mathbf{K}^{-1} \mathbf{c}\right)\right) \\
\mathbf{g}_{\mathbf{1}, \mathbf{1}}:=-\left(\frac{1}{2 \gamma} \mathbf{K}_{\mathbf{y}_{1} \mathbf{y}_{1}}^{-\mathbf{1}}\left(\mathbf{I}-\mathbf{K}_{\mathbf{y}_{1} \mathbf{y}_{2}} \mathbf{K}^{-1} \mathbf{K}_{\mathbf{y}_{2} \mathbf{y}_{1}} \mathbf{K}_{\mathbf{y}_{1} \mathbf{y}_{1}}^{-1}\right) \mathbf{e}\right) \\
\mathbf{g}_{1,2}:=\frac{1}{2 \gamma} \mathbf{K}_{\mathbf{y}_{1} \mathbf{y}_{1}}^{-1} \mathbf{K}_{\mathbf{y}_{1} \mathbf{y}_{2}} \mathbf{K}^{-1} \mathbf{e}
\end{array}
$$

Substituting (19) and (21) to (14) and (15), a solution for $\lambda_{1}$ and $\lambda_{2}$ is obtained:

$$
\left[\begin{array}{c}
\lambda_{1} \\
\lambda_{2}
\end{array}\right]=\left[\begin{array}{ll}
\mathbf{g}_{1,1}^{\mathbf{T}} \mathbf{e} & \mathbf{g}_{\mathbf{1}, \mathbf{2}}^{\mathbf{T}} \mathbf{e} \\
\mathbf{g}_{\mathbf{2}, \mathbf{1}}^{\mathbf{T}} \mathbf{e} & \mathbf{g}_{\mathbf{2}, \mathbf{2}}^{\mathbf{T}} \mathbf{e}
\end{array}\right]^{-1}\left[\begin{array}{c}
\alpha_{1}-\mathbf{g}_{\mathbf{1}, \mathbf{0}}^{\mathbf{T}} \mathbf{e} \\
\alpha_{2}-\mathbf{g}_{\mathbf{2}, \mathbf{0}}^{\mathbf{T}} \mathbf{e}
\end{array}\right]
$$

\section{EXPERIMENTS}

The performance of the algorithm is tested on two different kind of images: Images recorded with a standard camera (Lena and Checkboard) and microscopical images (Drosophila egg). These images have been chosen since they contain highly structural information like hair, chessboard pattern, single cells. In our experiments three parameters are varied in order to explore their influence on the restoration quality: standard deviation of Gaussian noise, standard deviation of Gaussian blur and the inverse filter size.

\section{A. Implementation}

The filter is implemented pixelwize, for a neighborhood of size $K \times K$ (in this implementation $K=35$ ). The covariance matrix $\mathbf{c}_{\mathbf{x y}}$ as well as the source variance $\sigma_{x}$ is estimated by the method described in [6]. Computing $\lambda_{i}$ is not necessary by a simple trick: For each neighborhood $n_{i}$ the mean $m_{i}$ is subtracted before applying the filter $g_{i}$. After the reconstruction the mean of the mean values $\left(\bar{m}=\frac{1}{N} \sum_{i=1}^{N} m_{i}\right)$ is added back to the reconstructed pixel. The computation of $\lambda_{i}$ can be omitted, since for zero-mean blocks the first quotient in the StatSSIM equation (8) is no longer dependend on g. Two-channels are used for the image reconstruction.

The algorithm is implemented in Matlab R2009a and the computation time for images of size $50 \times 50$ pixels is 30 seconds on a Intel Core 2 Duo processor with $3 \mathrm{GHz}$.

In Figure 2 the reconstruction of one part of the hair in the image Lena degraded by $\sigma_{\mathbf{h}_{1}}=1, \sigma_{\mathbf{h}_{2}}=2$ and $\sigma_{\eta}=5$ is presented.

The fine structure of the hair is recovered very nicely using the multi-channel approach. 

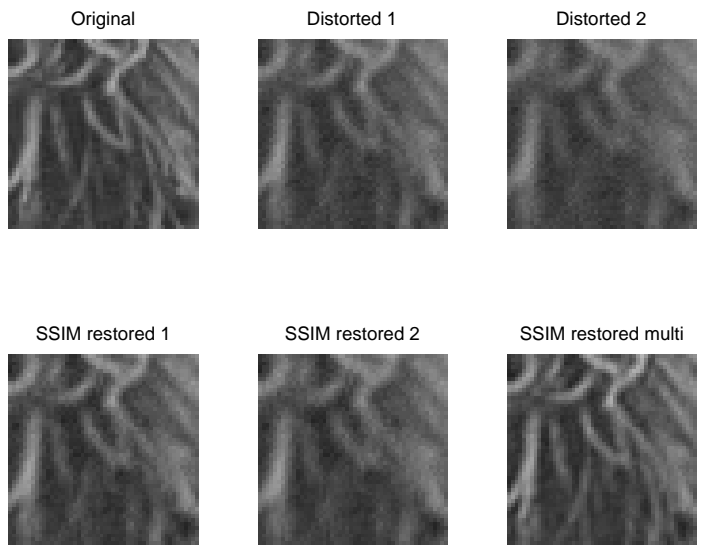

Figure 2. The original lena image (top row left) is distorted by $\sigma_{\eta}=5$, $\sigma_{\mathbf{h}_{1}}=1$ and $\sigma_{\mathbf{h}_{\mathbf{2}}}=2$ resulting in the images Distorted 1 (MSE: 102.128, SSIM: 0.917) and Distorted 2 ( MSE: 180.337, SSIM: 0.846) (top row middle and right). After SSIM restoration is applied the results are presented for single-channel restoration of Distorted 1 (MSE: 85.277, SSIM: 0.935) and Distorted 2 (MSE: 156.397, SSIM: 0.879)(bottom row left and middle) and multi-channel restoration (MSE: 52.673, SSIM: 0.962) (bottom row right)

\section{B. Influence of Noise}

The original image $\mathbf{x}$ is degraded with two gaussian blurs $\mathbf{h}_{1}$ and $\mathbf{h}_{2}$ resulting in two distorted images $\mathbf{y}_{1}$ and $\mathbf{y}_{2}$. The size of the blur is $W=5$ and the standard deviation (STD) of the blur is $\sigma_{\mathbf{h}_{1}}=1$ and $\sigma_{\mathbf{h}_{2}}=2$ respectively. The STD of the noise $\sigma_{\eta}$ is increased gradually from 0 to 50 pixels. Noise with same $\sigma_{\eta}$ is added to each blurred image, however the noise is generated for each image separately. The inverse filter length is $F=9$ pixels.

In Figure 3 you can see the SSIM values for different $\sigma_{\eta}$ and the reconstruction with two separately estimated inverse filters $\mathbf{g}_{1}$ and $\mathbf{g}_{2}$ (single im1 and single im2) and with the two combined inverse filters $\mathrm{g}$ (multi restaured). For $\sigma_{\eta}<10$, the multiview reconstruction does not improve the results. However, for $\sigma_{\eta}>$ 10 the multiview reconstruction improves the results significantly! Even for high noise $\left(\sigma_{\eta}=50\right)$, a high SSIM value can be reached.

In Figure 4 the results on the drosophila egg depicting single cells are shown. The parameter setting is the same as for the chessboard image. The cell structure can be recovered very well: even for $\sigma_{\eta}=0$ the multi-channel reconstruction exceeds the single-channel reconstruction.

\section{Influence of Blur}

For the chessboard image, the noise is kept constant $\sigma_{\eta}=30$ and vary $\sigma_{\mathbf{h}_{1}}$ and $\sigma_{\mathbf{h}_{2}}$, where $\sigma_{\mathbf{h}_{2}}=2 \sigma_{\mathbf{h}_{1}}$. From Figure 5 it is obvious that the SSIM multiview reconstruction is better than the single SSIM reconstructions, however as the blur increases, not much improvement can be achieved by using SSIM multiview reconstruction.

\section{Influence of Filter Size}

The SSIM value of the reconstructed images is explored for varying the filter size $F$. From Figure 6 the optimal filter size is 5 pixels, thus the reconstruction can be computed very quickly.

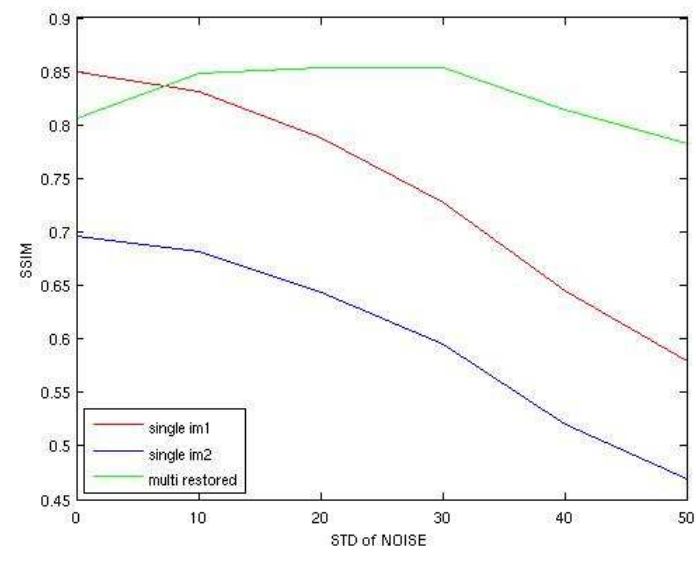

(a)
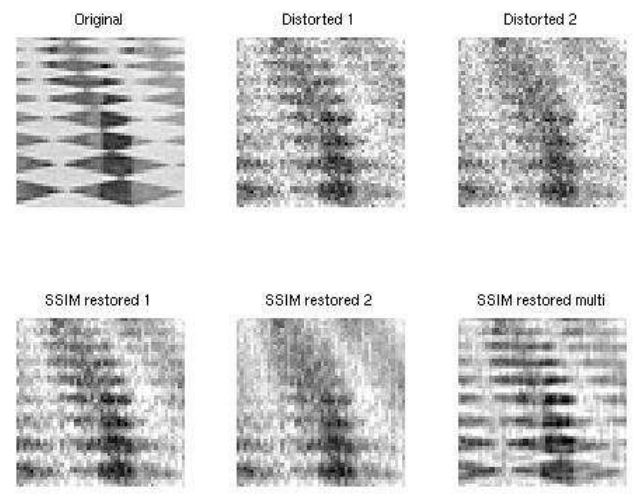

(b)

Figure 3. Influence of Noise on Checkboard. (a)The influence of noise (x-axis) on the SSIM index (y-axis) is plotted for single-channel restoration (red and blue) and multi-channel restoration (green). (b) The original chessboard image (top row left) is distorted by $\sigma_{\eta}=30, \sigma_{\mathbf{h}_{1}}=1$ and $\sigma_{\mathbf{h}_{2}}=2$ resulting in the images Distorted 1 (MSE: 1616.418, SSIM: 0.712) and Distorted 2 (MSE: 2266.447, SSIM: 0.572) (top row middle and right). After SSIM restoration is applied the results are presented for singlechannel restoration for Distorted 1 (MSE: 1371.034, SSIM: 0.745) and Distorted 2 (MSE: 1867.621, SSIM: 0.621) (bottom row left and middle) and multi-channel restoration (MSE: 692.040, SSIM: 0.861) (bottom row right).

\section{CONCLUSION}

The multi-channel SSIM deconvolution significantly improves the single-channel SSIM deconvolution. The method is in particularly very effective if the noise level is high. A small filter size is sufficient to achieve optimal reconstruction results.

Future research aims at extending this method to three dimensional microscopy images and applying it to data as described in [5]. Further the influence of the number of distorted images $M$ on the reconstruction quality will be studied.

\section{ACKNOWLEDGMENT}

The authors would like to thank S. S. Channappayya for providing the matlab code implementing the method described in [3]. This 


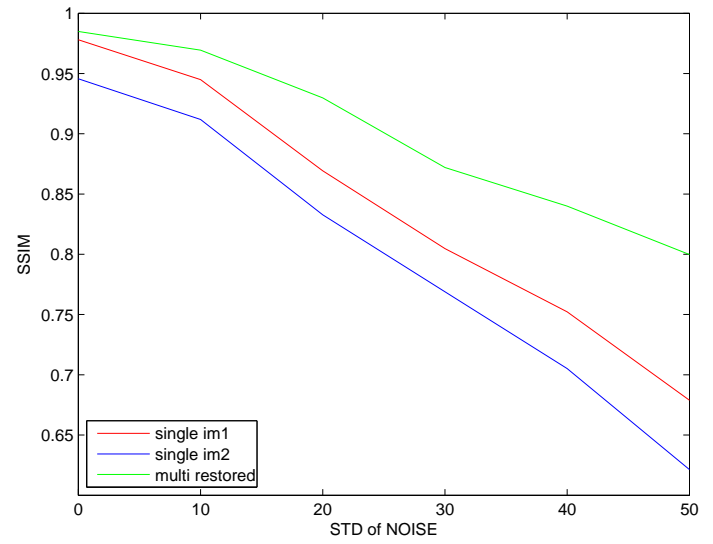

(a)
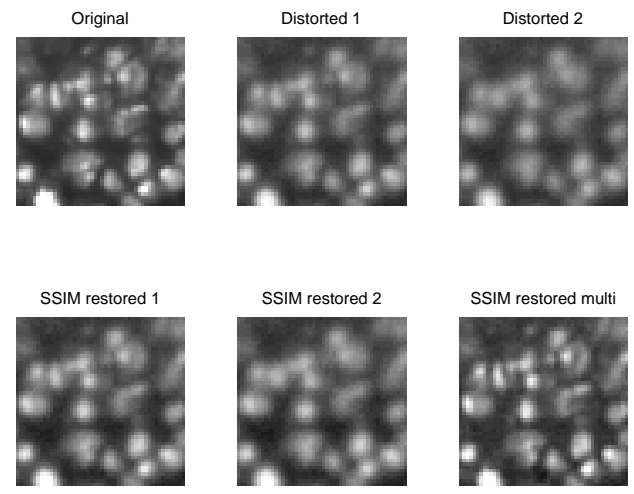

(b)

Figure 4. Influence of Noise on Drosophila Cells. (a)The influence of noise (x-axis) on the SSIM index (y-axis) is plotted for single-channel restoration (red and blue) and multi-channel restoration (green). (b) The original image (top row left) is distorted by $\sigma_{\eta}=3, \sigma_{\mathbf{h}_{1}}=1$ and $\sigma_{\mathbf{h}_{2}}=$ 2 resulting in the images Distorted 1 (MSE: 116.475, SSIM: 0.961) and Distorted 2 (MSE: 256.474, SSIM: 0.910) (top row middle and right). After SSIM restoration is applied the results are presented for single-channel restoration of Distorted 1 (MSE: 83.431, SSIM: 0.974) and Distorted 2 (MSE: 188.594, SSIM: 0.942) (bottom row left and middle) and multichannel restoration (MSE: 49.611, SSIM: 0.984) (bottom row right).

study was supported by the Excellence Initiative of the German Federal Governments (EXC 294) and the SFB 592.

\section{REFERENCES}

[1] Z. Wang, A. C. Bovik, H. R. Sheikh and E. P. Simoncelli Image Quality Assessment: From Error Visibility to Structural Similarity, IEEE Trans. Image. Process., vol.13, no.4, pp.600-612, Apr.2004.

[2] Z. Wang and A. C. Bovik, Mean Squared Error: Love It or Leave It?, IEEE Signal Process. Magazine, vol.26, no.1, pp.98-117, Jan.2009.

[3] S. S. Channappayya, A. C. Bovik, C. Ceramanis and R. W. Heath, Design of Linear Equalizers Optimized for the Structural Similarity Index, IEEE Trans. Image. Process., vol.17, no.6, pp. 857-872, 2008.

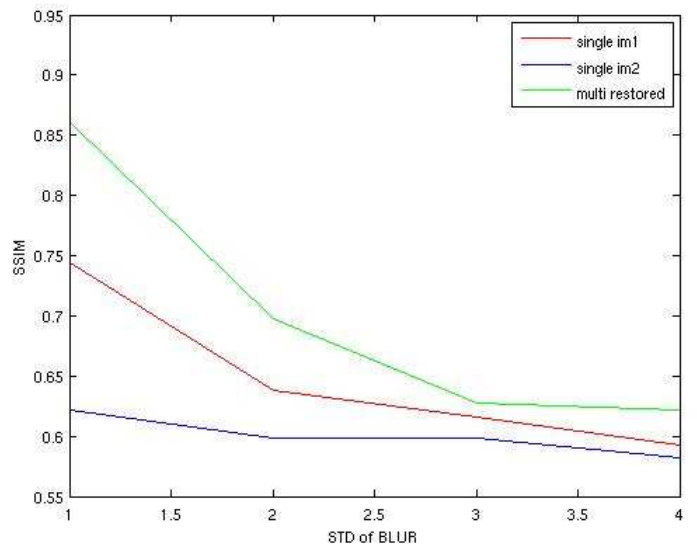

Figure 5. Influence of Blur size. The SSIM values of the single-channel restored chessboard image and the multi-channel restored chessboard image (y-axis) are plotted against the blur standard deviation $\sigma_{\mathbf{h}_{1}}$ with $\sigma_{\mathbf{h}_{2}}=$ $2 \sigma_{\mathbf{h}_{1}}$ (x-axis).

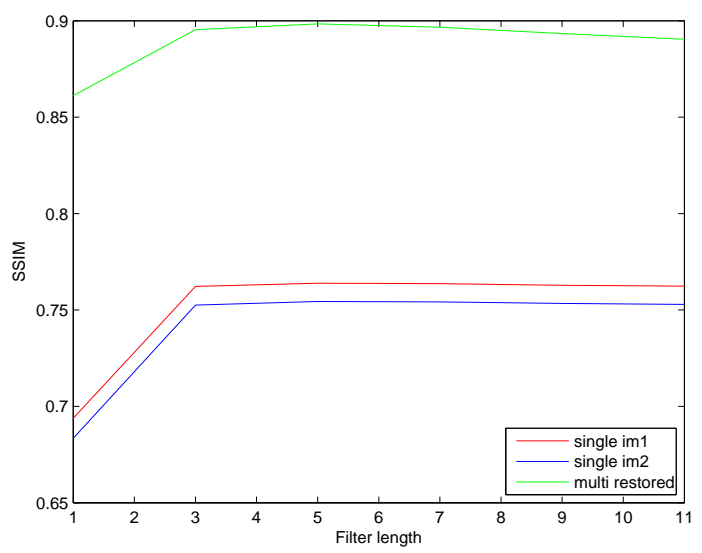

Figure 6. Influence of Filter size. The SSIM values of the singlechannel restored image and the multi-channel restored image (y-axis) are plotted against the filter size (x-axis). Here results for the lena image with parameters $\sigma_{\mathbf{h}_{\mathbf{1}}}=3, \sigma_{\mathbf{h}_{\mathbf{2}}}=6$ and $\sigma_{\eta}=15$ is presented.

[4] F. Sroubek and J. Flusser, Multichannel Blind Iterative Image Restoration, IEEE Trans. Image. Process., vol.12, no.9, pp. 1094-1106, Sep.2003.

[5] P. J. Verveer and T. M. Jovin Improved Restoration from Multiple Images of a Single Object: Application to Fluorescence Microscopy, Applied Optics, vol.37, no.26, pp. 6240-6246, 1998.

[6] J. Portilla and E. Simoncelli, Image restoration using Gaussian scale mixtures in the wavelet domain, in Proc. IEEE Intl Conf. on Image Proc., pp. 965-968, 2005.

[7] R. C. Hardie, K. J. Barnard and E. E. Armstrong Joint MAP registration and highresolution image estimation using a sequence of undersampled images, IEEE Trans. Image. Process., vol.6, pp. 1621-1633, 1997. 